\title{
SN1987A AND CONSTRAINT ON THE MASS AND LIFETIME OF TAU NEUTRINOS
}

\author{
Mariko TAKAHARA ${ }^{a}$ and Katsuhiko SATO $^{b}$
}

Department of Physics, Tokyo Institute of Technology, Tokyo, Japan

$b$ Departments of Physics, University of Tokyo, Tokyo, Japan

Recently it has been suggested that neutrinos have nonvanishing mass from both experimental and theoretical investigations. Accelerator experiments and cosmology, however, impose the constraints on mass $\mathrm{m}_{\nu}$ and lifetime $\tau_{\nu}$ of $\nu_{\tau}$ (see Fig. 1). As we showed in the previous paper [1], supernova explosion also imposes the stringent constraint on them. The purpose of this paper is to investigate the constraint imposed on $\mathrm{m}_{\nu}$ and $\tau_{\nu}$ of $\nu_{\tau}$ from SN1987A.

Tau neutrinos are emitted from the central part of the iron core together with the electron neutrinos because they are also in thermal equilibrium with matter there. When tau neutrinos decay on the way of streaming out through the decay modes of $\nu_{\tau} \rightarrow \nu_{e}+\gamma$ and $\nu_{\tau} \rightarrow e^{+}+e^{-}+\nu_{e}$, $\gamma-$ rays are produced in both cases (in the latter case, the positron annihilates in the ambient matter and produces $\gamma$-rays).

If tau neutrinos emitted from SN1987A decayed outside the star, the $\gamma$-ray burst following the $\nu$-burst could be expected. Therefore observation of the $r$-ray burst from SN1987A imposes a strict constraint on the mass and lifetime of tau neutrinos.

We estimated the $\gamma$-ray flux produced by the decay mode of $\nu_{\tau} \rightarrow \nu_{e}+\gamma$ because it is difficult to estimate the $\gamma$-ray flux produced by the decay mode of $\nu_{\tau} \rightarrow \nu_{e}+e^{+}+e^{-}$due to the ambiguity of the amount of the matter which surrounded the progenitor of SN1987A.

We assumed that tau neutrinos are emitted from their neutrinosphere with the spectrum of Fermi-Dirac distribution with zero chemical potential determined by the temperature at the neutrinosphere. This temperature is estimated to be $5 \mathrm{MeV}$ from the spectrum calculated by wilson and his collaborators.

The radius of the neutrinosphere and duration time of neutrino emission are adjusted so that the total energy lost by neutrinos should be the binding energy of neutron stars $3 \times 10^{53} \mathrm{erg}$.

If $\tau_{\nu}$ is much longer than the time scale of streaming out $t_{0}$, it is assumed that tau neutrinos decay outside the star at the typical distance from the center of the star $R=v \tau_{\nu} /\left(1-v^{2} / c^{2}\right)^{1 / 2}(v$ and $c$ denote the velocity of electron neutrinos and light, respectively) and 
that the energy of $\nu_{\tau}$ is shared equally between $\gamma$-ray and $\nu_{e}$. The distance to $\mathrm{SN} 1987 \mathrm{~A}$ is assumed to be $48 \mathrm{kpc}$. It is to be noticed that the arrival time of $\gamma$-rays spreads to the order of $2 \mathrm{R} / \mathrm{c}$.

On the other hand, if $\tau_{\nu}<<t_{0}$, almost all neutrinos decay inside the star. Therefore in this case r-rays are produced at the surface of the star and $\gamma$-ray burst spreads to the time scale of $2 t_{0}$. As for the details of the calculation of the $\gamma$-ray flux, see Ref. [2].

At present the $\gamma$-ray detectors in operation are those aboard the Solar Maximum Mission (SMM) and Pioneer Venus Orbiter (PVO). We estimate the typical detection limit of the counter of SMM to be 0.1 counts/s/cm and that of PVO 10 counts/s/cm ${ }^{2}$, respectively. Since the evidence of $\gamma$-ray burst from $S N 1987 \mathrm{~A}$ has not been reported yet, we investigated the constraint on $\mathrm{m}_{\nu}$ and ${ }^{\gamma}{ }_{\nu}$ of $\nu_{\tau}$ on the assumption that the r-ray burst was not observed.

In Fig. 1 we showed the prohibited region of the $m_{\nu}$ and $\tau_{\nu}$ of $\nu_{\tau}$ by the horizontally hatched regions (SMM) and dashed lines (PVO) for the various radii of the progenitor of $S N 1987 \mathrm{~A}, \mathrm{R}_{0}$.

The region prohibited by the experiment, cosmology and $\gamma-$ ray background radiation from the supernovae are also shown in Fig. 1 by the dotted region, the vertically hatched region, and obliquely hatched region, respectively.

Comparing these constraints with that imposed by the present paper, it is obviously seen that the region allowed by the previous constraints $\left(\mathrm{m}_{\nu} \sim 50 \mathrm{MeV}, \tau_{\nu} \sim 10^{3} \mathrm{sec}\right)$ is completely prohibited by the present constraint one if $R_{0}<10^{13} \mathrm{~cm}$, which is supported by the observation of the light curve of $\mathrm{SN} 1987 \mathrm{~A}$.

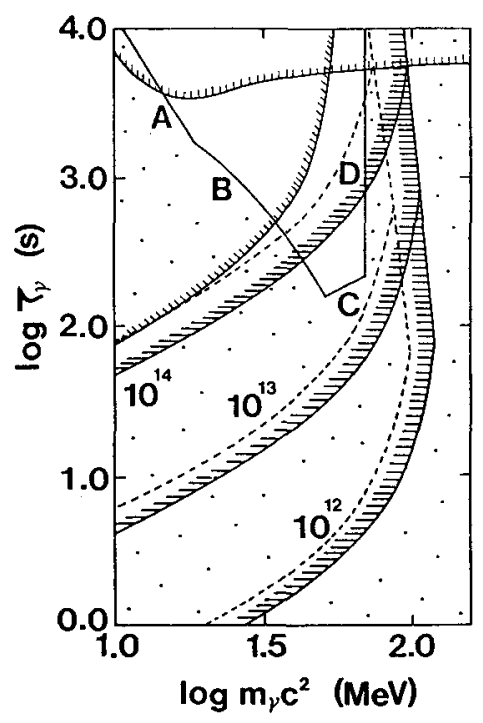

Fig. 1 Constraints on mass and lifetime of tau neutrinos. For details, see in the text.

[1] M. Takahara and K. Sato, Phys. Lett. 174B (1986) 373 .

[2] M. Takahara and K. Sato, Modern Phys. Lett., A2 (1987) 293. 